\section{Sampling, prevalence and characterization of methicillin-resistant Staphylococcus aureus on two
Belgian pig farms}

\author{
Isabelle Dewaele, ${ }^{1}$ Winy Messens, ${ }^{1}$ \\ Ingrid De Man,2 Pierre Delputte,2 \\ Lieve Herman,1 Patrick Butaye,3,4 \\ Marc Heyndrickx, ${ }^{1}$ Geertrui Rasschaert ${ }^{1}$ \\ 1Technology and Food Science Unit, \\ Institute for Agricultural and Fisheries \\ Research (ILVO), Melle; 2Department \\ HIVB, Catholic University College South- \\ West-Flanders, Roeselare; ${ }^{3}$ Veterinary \\ and Agrochemical Research Centre, \\ Brussels; ${ }^{4}$ Department of Pathology, \\ Bacteriology and Poultry Diseases, \\ Faculty of Veterinary Medicine, Ghent \\ University, Merelbeke, Belgium
}

\section{Abstract}

This study investigated the spread of MRSA (methicillin-resistant Staphylococcus aureus) on two Belgian pig farms. Pigs of different ages (from farrowing to slaughter age and sows) as well as the barn environment were screened extensively on two occasions three months apart. A subset of MRSA isolates was tested for antimicrobial susceptibility to 16 antibiotics and was further characterized by pulsed-field gel electrophoresis. Ninety-five percent and $77 \%$ of the tested pigs on farm $\mathrm{A}$ and farm B, respectively, were colonized with MRSA. MRSA positive animals were detected in all age categories sampled on each sampling day. Piglets were already colonized in the farrowing unit with the same or other MRSA strains than their mother. The prevalence of MRSA colonized pigs increased significantly after weaning and decreased during the fattening period. Pigs carried MRSA mainly in the nares, followed by the perineum and skin and to a lesser degree the rectum. A pig could be contaminated or colonized with different MRSA strains at the same time. The barn environment was also found to be contaminated with different MRSA strains, including the air inlet and outlet. All isolates tested on both farms were resistant to both tetracycline and trimethoprim, while they were susceptible to rifampicin, mupirocin and linezolid. There was a significant difference in resistance prevalence between the two farms for the antibiotics gentamicin, kanamycin, tobramycin, tylosin, lincomycin and quinupristin/dalfopristin. Furthermore, several antibiotic resistance profiles were observed within one farm.
This study clearly indicates that several MRSA strains circulate on one farm, from the nursery unit to the fattening unit. This is important to consider when attempts are made to remediate these farms.

\section{Introduction}

Methicillin-resistant Staphylococcus aureus (MRSA) is an important human pathogen responsible for a wide spectrum of diseases, ranging from relatively harmless skin infections to life-threatening illnesses.1,2 Until recently, two different MRSA types were reported, namely hospital-acquired MRSA (HAMRSA) and community-acquired MRSA (CAMRSA). ${ }^{3,4}$ A few years ago, a new MRSA type was described, i.e. the livestock-associated MRSA (LA-MRSA). ${ }^{5-7}$ In the Netherlands, this MRSA type has been reported at high frequency among pigs and pig farmers. ${ }^{2,5}$ The LA-MRSA type has also been isolated from several other livestock animals such as cattle, horses and poultry. ${ }^{8-10}$ A survey of 50 Belgian pig farms has shown that $68 \%$ of the farms and $44 \%$ of the pigs tested positive for MRSA. 11 This study indicates that MRSA contamination is wide spread in Belgian pig husbandry. Several factors may play an important role in the dissemination of MRSA on the pig farm, to other pig farms and to the community. Pig to human transmission is possible.5,12,13 MRSA carriage is significantly higher among veterinarians than people who are not in contact with animals professionally. ${ }^{14}$ Because veterinarians visit various farms, they may play an important role in spreading MRSA to and among pig farms. MRSA has already been isolated from environmental surfaces $^{15}$ and has a strong ability to survive in dust. ${ }^{16}$ In this respect, it is possible that MRSA can spread from animals to the barn environment and vice versa.

The objectives of the present study were i) to determine the distribution of MRSA colonized pigs on two MRSA-positive pig farms, ii) to study if MRSA carriage of the pigs is agerelated, iii) to evaluate different sampling sites to determine the MRSA status of a pig, iv) to examine the MRSA contamination of the barn environment, and finally v) to determine the population variability of the MRSA by an antibiotic resistance profile and pulsed field gel electrophoresis (PFGE).

\section{Materials and Methods}

\section{Survey on pig farms and sample collection}

Two Belgian pig farms (FARM) were exten-
Correspondence: Rasschaert Geertrui, Institute for Agricultural and Fisheries Research (ILVO), Technology and Food Science Unit, Brusselsesteenweg 370, B-9090 Melle, Belgium.

Tel. +32.9.2723026 - Fax: +32.9.272300.

E-mail: geertrui.rasschaer@@ilvo.vlaanderen.be

Key words: methicillin-resistant Staphylococcus aureus, pigs, age group, antibiotic profile, pulsed field gel electrophoresis.

Acknowledgements: the authors would like to thank the pig farmers who participated in this study and assisted with taking sample and Arnold Stael, DVM, who took samples. They would also thank Nika Janež and Evy Maes for technical assistance and Miriam Levenson for critical reading of the manuscript. This study was funded by the Flemish Government.

Contributions: ID, sample collection, isolation, phenotypic detection and genotypic detection of MRSA, antimicrobial susceptibility testing, analysis and interpretation of data, drafting and final approval; WM, statistical analysis of data, drafting the article, final approval; IDM, sample collection, design of study, drafting and final approval; PD, $\mathrm{LH}, \mathrm{PB}, \mathrm{MH}$, design of study, revising the article and final approval; GR, sample collection, PFGE, analysis and interpretation of data, drafting and final approval.

Received for publication: 8 October 2010 . Accepted for publication: 10 February 2011.

Conflict of interest: the authors report no conflicts of interest.

This work is licensed under a Creative Commons Attribution 3.0 License (by-nc 3.0).

(C) Copyright I. Dewaele et al., 2010

Licensee PAGEPress, Italy

Veterinary Science Development 2011; 1:e1 doi:10.4081/vsd.2011.e1

sively screened for MRSA presence at two sampling times (SAMPLING TIME). The first sampling was carried out in the period from April May 2007, while the second sampling was done about three months later (August-September 2007). Farm A is a farrow-to-finish farm, a socalled closed farm where no pigs are purchased. Farm B is an open breeding farm, where the piglets are born on the farm but are later transported to various fattening pig farms. Samples were collected from sows and from pigs of different age groups (PIG GROUP). On farm A, six age groups, i.e. 1-4 weeks, 4-8 weeks, 8-12 weeks, 12-16 weeks, 16 20 weeks, 20-26 weeks, as well as sows were sampled. On farm B, three age groups, i.e. 1-4 weeks, 4-8 weeks, $8-2$ weeks, as well as sows were sampled. The piglets of age group 1 to 4 weeks were housed together with the sows in the nursery unit. 
Between six and eight pigs of each age group were randomly selected. For the sows, usually more animals were sampled, especially in farm B, where the pig population consisted mainly of sows. Each pig was sampled using a nasal, skin (behind the ear as this is often one of the cleanest zones of the pig), perineal and rectal swab. In addition to sampling the pigs, the environment of the barns was sampled (samples) by taking swabs of the floor, wall, drinking nipples and ventilation system (inlet and outlet) in a pen of each age group. Samples were taken using swabs pre-moistened with sterile physiological water. In addition, air samples at the ventilation (inlet and outlet) were taken in the stables using an air sampler (Biotest AG, Dreieich, Germany). Each time, 100 litres of air was sampled. The air strips were made with 0xacillin Resistance Screening Agar Base (ORSAB, CM1008, 0xoid, Basingstoke, UK). Finally, the pig farmer and his family were sampled using a nose swab.

The pig farmers were questioned concerning the antibiotic use. A list was viewed on the antibiotic group treatments performed on the farm in the past half year before the samplings started.

\section{Isolation and phenotypic detection of methicillin-resistant}

\section{Staphylococcus aureus}

Swabs were placed in Brain Heart Infusion (BHI, CM0225, 0xoid) supplemented with $7.5 \%(\mathrm{w} / \mathrm{v}) \mathrm{NaCl}$. After $24 \mathrm{~h}$ incubation at $37^{\circ} \mathrm{C}$, a loopful of the broth was sub-cultured for another 24 to $48 \mathrm{~h}$ at $37^{\circ} \mathrm{C}$ on selective chromogenic agar for MRSA (ChromID MRSA, 43459, Biomérieux, Marcy l'Etoile, France). Two typical green colonies were picked up and subcultured on BHI agar plates (CM0375, 0xoid). The air strips (Biotest AG) were incubated for $24 \mathrm{~h}$ at $37^{\circ} \mathrm{C}$. From each strip, three typical blue colonies were picked up and subcultured on BHI agar plates.

\section{Genotypic identification of methi- cillin-resistant Staphylococcus aureus}

DNA was extracted from suspected isolates as described by Strandén et al.,17 with minor modifications. Briefly, cells were harvested from BHI agar plates and resuspended in $50 \mu \mathrm{L}$ of lysostaphin $(100 \mu \mathrm{g} / \mathrm{mL})$, followed by incubation for $10 \mathrm{~min}$ at $37^{\circ} \mathrm{C}$. Subsequently, $5 \mu \mathrm{L}$ proteinase $\mathrm{K}$ solution $(2.5 \mathrm{mg} / \mathrm{mL})$ and $150 \mu \mathrm{L}$ $0.1 \mathrm{M}$ Tris-HCl (pH 8) were added, followed by incubation for $10 \mathrm{~min}$ at $60^{\circ} \mathrm{C}$ and then $5 \mathrm{~min}$ at $100^{\circ} \mathrm{C}$. Cell lysates were stored at $-20^{\circ} \mathrm{C}$ until further use.

A multiplex PCR 18 amplifying target sequences mecA, nuc and 16S rRNA was applied to confirm the isolates as MRSA. The following reference strains were used as posi- tive and negative controls: ATCC 33592 (MRSA), ATCC 43300 (MRSA) and ATCC 25923 (MSSA, methicillin-sensitive Staphylococcus aureus).

\section{Antimicrobial susceptibility testing of methicillin-resistant}

\section{Staphylococcus aureus isolates}

Susceptibility was determined using the Kirby-Bauer disk-diffusion method according to the guidelines of Rosco and Rosco tablets (http://rosco.dk). A selection of 140 pig isolates were tested covering both farms, both sampling days and all age groups. Subsequently, sixteen antibiotics were tested with Neo-sensitabs $^{\mathrm{TM}}$ (Rosco, Taastrup, Denmark): chloramphenicol $(60 \mu \mathrm{g})$, ciprofloxacin $(10 \mu \mathrm{g})$, erythromycin $(78 \mu \mathrm{g})$, fucidin $(100 \mu \mathrm{g})$, gentamicin $(40 \mu \mathrm{g})$, kanamycin $(100 \mu \mathrm{g})$, lincomycin $(19 \mu \mathrm{g})$, linezolid $(30 \mu \mathrm{g})$, mupirocin $(10 \mu \mathrm{g})$, quinupristin/dalfopristin $(15 \mu \mathrm{g})$, rifampicin $(30 \mu \mathrm{g})$, sulphonamide $(240 \mu \mathrm{g})$, tetracycline $(80 \mu \mathrm{g})$, tobramycin $(40 \mu \mathrm{g})$, trimethoprim $(5.2 \mu \mathrm{g})$ and tylosin $(150 \mu \mathrm{g})$. Mueller-Hinton plates (Oxoid, CM0405, Basingstoke, UK) were inoculated with the isolates, following incubation with Neo-sensitabs $^{\mathrm{TM}}$ at $37^{\circ} \mathrm{C}$. Plates were read after $24 \mathrm{~h}$. ATCC 25923 (MSSA) was included for internal quality control.

\section{Characterization of methicillin-resist-} ant Staphylococcus aureus isolates by pulsed field gel electrophoresis

Thirty-seven selected MRSA isolates were typed using PFGE with SmaI, SacII and ApaI restriction enzymes as described by Rasschaert et al. ${ }^{19}$ These isolates were chosen based on the obtained antibiotic resistance profiles and covered both farms, all sampling days and all age groups. Gel patterns were analyzed using BioNumerics version 5.10 (Applied Maths, Sint-Martens-Latem, Belgium) and clustered using Dice coefficient (tolerance 0.5$1.5 \%$, optimization $1 \%$ ) and the unweighted pair group method using averages (UPGMA). A consensus matrix, including combined PFGE patterns of both restriction enzymes, was constructed and a cut-off value of $97 \%$ was used to discriminate between different pulsotypes. Pulsotypes were designated by a combination of the name of the restriction enzymes followed by a pulsotype number, e.g., Sac1/Apal.

\section{Statistical analysis}

The P-value of the odds ratios (OR) was calculated according to the Fisher's exact test. For each farm separately, the effect of the categorical predictors PIG GROUP, SAMPLE and SAMPLING TIME on the probability of observing a positive MRSA sample was analyzed using a logistic regression model. Although we were not particularly interested in the effect of SAMPLING TIME, this predictor was included in the model to account for this effect. A main effects model was chosen, because the interaction term PIG GROUP*SAMPLE was not significant. The significance level $\alpha$ was set at 0.05 . All statistical analyses were performed using $\mathrm{R}$ version 2.7.2 (2008-08-25).

A pig was considered as MRSA colonized if at least one of the four swabs was positive. The total number of MRSA colonized pigs was used to calculate the detection rate of each of the four sampling places: nares, perineum, skin or rectum. The number of MRSA positive pigs obtained by (for example) taking only nasal swabs was divided by the total number of MRSA colonized pigs. This was repeated for the other three sampling places and the combination of two sampling places. For each sampling site the $95 \%$ confidence interval (CI) was calculated using $\mathrm{R}$ version 2.7.2.

\section{Results}

\section{Prevalence of methicillin-resistant}

Staphylococcus aureus on the two pig farms

On farm A, MRSA was not detected in the pig farmer and his family at the time of sampling. Both the pig farmer and his wife on farm B were MRSA nasal carriers, whereas the three children were free of MRSA.

MRSA positive pigs were detected within all groups of pigs sampled in both farms and at both sampling times (Table 1). The prevalence of colonized pigs was very high on farm A, $90 \%$ $(\mathrm{n}=50)$ on the first sampling day and $99 \%$ $(n=59)$ at the second sampling day. The prevalence of pigs carrying MRSA found on farm B was lower: $84 \%(n=49)$ on the first sampling day and $55 \%(n=49)$ on the second sampling day. MRSA positive animals were detected in all sampled age categories at each sampling day. The barn environment was also contaminated on both farms (Table 1). On farm A, MRSA was found in $63 \%(\mathrm{n}=47)$ and $55 \%$ $(n=60)$ of the barn environment samples, for the first sampling and the second sampling day, respectively. For farm B, the prevalence of contaminated environmental samples was again lower: $62 \%(n=43)$ on the first sampling day and $40 \%(n=8)$ on the second sampling day. MRSA was detected on the floor, the wall, the drinking nipples and the ventilation system. In some pens, MRSA was also detected in the air. At farm A, the main effect model that was fitted to the data has demonstrated a significant effect on the prevalence of MRSA positive samples for PIG GROUP $(\mathrm{P}=0.0022)$ and SAMPLE $(\mathrm{P}<2.2 \mathrm{E}-16)$. The SAMPLING TIME was included in the model, but was not found 
to be significant $(\mathrm{P}>0.05)$. The interaction term PIG GROUP*SAMPLE was not significant $(\mathrm{P}>0.05)$ and was therefore not included in the model. For the reference category (pigs aged 1-4 weeks and sampling pigs), the number of successes was about 20.0 times (95\% CI: 5.03-101.9) higher than the number of failures. Significantly more pigs were positive at the age of 4-8 weeks and 8-12 weeks compared to pigs of age 16-20 weeks, $20-26$ weeks and sows with estimated odds ratios of 0.049 (95\% CI: 0.047-0.33), 0.066 (95\% CI: 0.0064-0.44) and 0.12 (95\% CI: $0.013-0.73$ ), respectively. The estimated odds ratio for samples taken when pigs were aged 4-8 weeks and 8-12 weeks versus $1-4$ weeks was 7.04 (95\% CI: 0.98-74.6) and 7.58 (95\% CI: 1.07-78.5), respectively, and were not significant $(\mathrm{P}=0.07$ and $\mathrm{P}=0.056$, respectively). When comparing the different sampling sites taken, the highest proportion of positive samples was detected on the floor, the pigs and the wall (Table 1). This is also visualised in the two upper graphs in Figure 1.

For farm B, the main effect model that was fitted to the data also has demonstrated a significant effect for PIG GROUP ( $\mathrm{P}=4.07 \mathrm{E}-12)$ and SAMPLE $(\mathrm{P}=1.53 \mathrm{E}-10)$. The SAMPLING TIME was included in the model and was significant $(\mathrm{P}=3.31 \mathrm{E}-05)$. The interaction term PIG GROUP*SAMPLE was not significant $(\mathrm{P}>0.05)$ and was not included in the model. For the reference category (pigs aged 1-4 weeks and sampling pigs), the number of successes was about 3.74 times $(95 \% \mathrm{CI}$ : 1.31 11.7) higher than the number of failures.

Sows were significantly less colonized than pigs aged 4-8 weeks and 8-12 weeks with estimated odds ratios of 0.005 (95\% CI: -8.705- $3.06)$ and 0.019 (95\% CI: 0.0009-0.12), respectively. The estimated odds ratio for samples taken when pigs were aged 4-8 weeks and 8-12 weeks vs. 1-4 weeks was 163.5 (95\% CI: 15.1$5328)$ and 42.9 (95\% CI: 5.92-922), respectively, and were highly significant $(\mathrm{P}<0.001$ and $\mathrm{P}<0.01$, respectively). When comparing the samples taken, the highest proportion of positive samples was detected on the nipples, the pigs and the wall, followed by the floor (Table $1)$. These results are shown in the two lower graphs of Figure 1.

Of all colonized pigs, MRSA was predominantly detected in the nares (detection rate, DR: 0.83$)$, followed by the perineum $(\mathrm{DR}=0.71)$ and skin $(\mathrm{DR}=0.69)$ and to a lesser degree in the rectum ( $\mathrm{DR}=0.47$ ) (Table 2).

Comparing skin and perineal swabs, there were no significant differences between these two sampling methods. When using a combination of pig sampling sites, the detection rate to identify a MRSA colonized pig will increase (for example, the combination nares and perineum with a sensitivity of 0.96 ).
Table 1. Number of methicillin-resistant Staphylococcus aureus positive samples in the different pig age groups and environment for two pig farms on two sampling days. In the first column, the pig group, sample or sampling day is given, in the second and third column data of farm $A$ and $B$ are given, respectively.

\section{Farm A Farm B}

$\begin{array}{lc}\text { Pig group } & \\ 1-4 \mathrm{w} & 20 / 27(14 / 14)^{*} \\ 4-8 \mathrm{w} & 24 / 27(14 / 14) \\ 8-12 \mathrm{w} & 25 / 28(14 / 14) \\ 12-16 \mathrm{w} & 27 / 35(14 / 14) \\ 16-20 \mathrm{w} & 17 / 28(11 / 14) \\ 20-26 \mathrm{w} & 19 / 29(13 / 15) \\ \text { Sow } & 37 / 49(23 / 24)\end{array}$

$12 / 27(7 / 14)$

$25 / 28(14 / 14)$

$23 / 28(14 / 14)$

NP

$\mathrm{NP}$

$\mathrm{NP}$

Sow

$44 / 106(33 / 56)$

\begin{tabular}{lcc} 
Sample & & \\
Air in & $1 / 15$ & $2 / 13$ \\
Air out & $6 / 15$ & $2 / 11$ \\
Floor & $17 / 18$ & $7 / 14$ \\
Nipples & $9 / 16$ & $8 / 14$ \\
Pigs & $103 / 109$ & $68 / 98$ \\
Ventilation in & $8 / 16$ & $1 / 13$ \\
Ventilation out & $10 / 16$ & $6 / 12$ \\
Wall & $15 / 18$ & $10 / 14$ \\
\hline Sampling day & & \\
Day 1 & $80 / 104$ & $63 / 92$ \\
Day 2 & $89 / 119$ & $41 / 97$ \\
\hline
\end{tabular}

$\mathrm{NP}$, age group not present on the farm; *total number of positive samples/total number of samples tested (number of positive pigs/total number of pigs tested).
FARM A

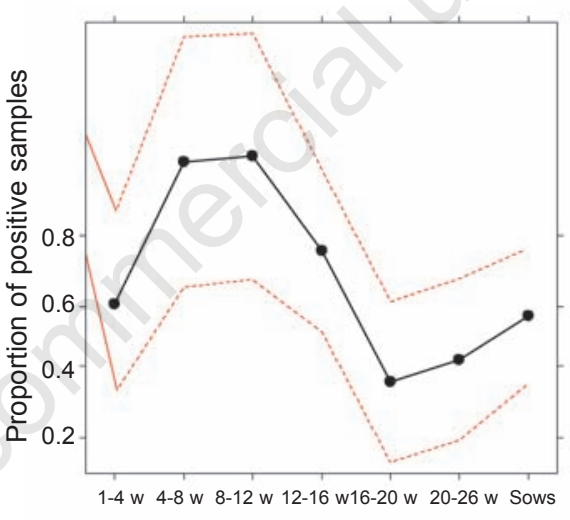

Pig group

FARM B

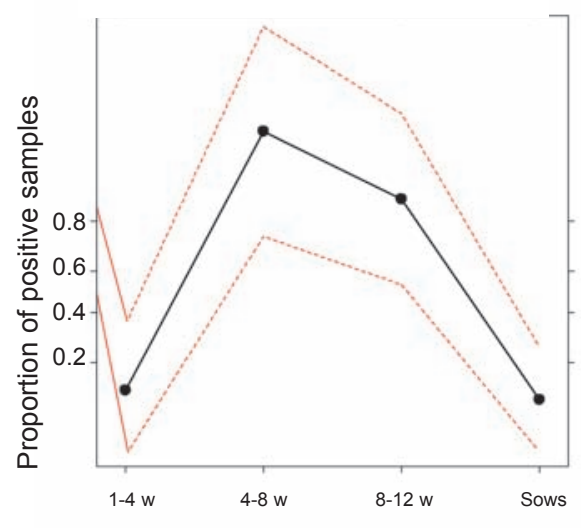

Pig group
FARM A

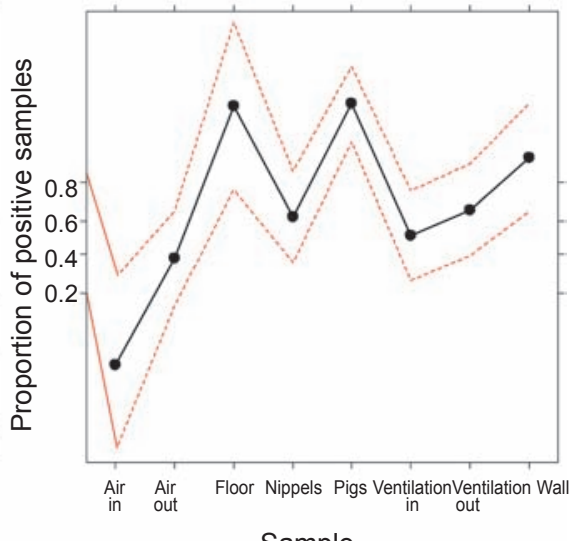

Sample

FARM B

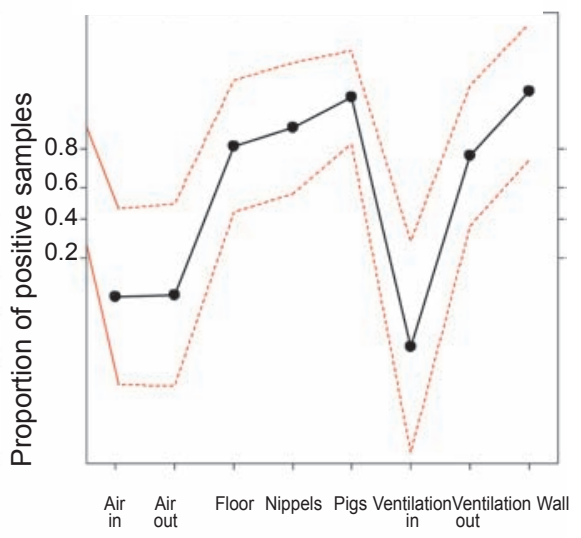

Sample
Figure 1. Estimated probability of observing a positive MRSA sample at farm A (above) and $B$ (below) as a function of Pig group and Sample. The effect function in the effects package of $R$ was used for this purpose. 


\section{Antimicrobial susceptibility}

The antimicrobial susceptibility was tested for 140 MRSA pig isolates. A summary of the antibiotic resistance of these pig isolates from both farms is given in Table 3 .

Overall, all tested isolates (of both farms) were resistant to both tetracycline and trimethoprim. They were all susceptible to rifampicin, mupirocin and linezolid and nearly all isolates were susceptible to chloramphenicol and fucidin. There was a significant difference in resistance prevalence between the two farms for the antibiotics gentamicin, kanamycin, tobramycin, tylosin, lincomycin and quinupristin/dalfopristin. While most of the isolates of farm B were resistant to gentamicin (97\%), kanamycin (94\%) and tobramycin (94\%), they were nearly all susceptible in farm A (7\%, 5.6\% and 5.6\%, respectively). Resistance to tylosin was $51 \%$ of the isolates for farm A compared to $70 \%$ for farm B. Nearly all the isolates (99\%) of farm A were resistant to lincomycin, compared to $70 \%$ of the isolates of farm B. Quinupristin/dalfopristin resistance was found in $32 \%$ of the isolates of farm A, compared to $7.5 \%$ for farm $B$. The MRSA isolate of the farmer from farm $B$ had a somewhat different antibiotic resistance profile on the second sampling than on the first. At the first sampling day, the MRSA isolate showed multiple resistance to tetracycline, trimethoprim, gentamicin, lincomycin, tylosin and erythromycin. At the second sampling, the isolate had an additional resistance to tobramycin and kanamycin. On both farms, the most frequent used antibiotics for group treatments were $\beta$-lactam antibiotics (amoxicillin, ampicillin, penicillin and ceftiofur) and fluoroquinolones (enrofloxacine). Tetracyclines, colistine and the combination of sulfadiazine with trimethoprim were also used on both farms, but only sporadically. Furthermore, on farm A, macrolides (tylosine, tilmicosine) were frequently used, which were not administered on farm B. On farm B, there was also sporadic use of lincomycine.

Table 2. The detection rate of each sampling method and combination of sampling methods, given with corresponding 95\% CI.

\begin{tabular}{lcc} 
& Detection rate & $95 \% \mathrm{CI}$ \\
Nares & 0.83 & $(0.77-0.88)$ \\
Perineum & 0.71 & $(0.64-0.78)$ \\
\hline Skin & 0.69 & $(0.61-0.76)$ \\
Rectum & 0.47 & $(0.39-0.54)$ \\
\hline Nares + Perineum & 0.96 & $(0.92-0.98)$ \\
Nares + Skin & 0.92 & $(0.87-0.96)$ \\
\hline Nares + Rectum & 0.89 & $(0.83-0.93)$ \\
Skin + Perineum & 0.88 & $(0.82-0.93)$ \\
\hline Skin + Rectum & 0.81 & $(0.75-0.87)$ \\
Perineum + Rectum & 0.79 & $(0.72-0.85)$ \\
\hline
\end{tabular}

\section{Characterization}

The isolates were not typeable by PFGE with restriction enzyme $S m a$, but they were all typeable by PFGE using the other two restriction enzymes. On both farms, a variety of different pulsotypes was found. On farm A, six different pulsotypes were distinguished: five groups with only one or two isolates each, and one group with 13 isolates (Sac1/Apa3; Figure 2). The latter group contained isolates from both the first sampling day and the second sampling day. On farm B, five different pulsotypes were distinguished. In analogy with farm A, one large group (Sac8/Apa8) was found covering both sampling days. In the two large groups on farm A and B, several antibiotic resistance profiles were determined (Figure 2). One pulsotype (Sac3/Apal) was isolated from both farms. These isolates also shared the same antibiotic profile. Two other pulsotypes; Sac1/Apa3 and Sac10/Apa9 detected on farm A and B, respectively, were very similar to each other.

From some pigs several isolates were found at the same sampling time. These isolates were not necessarily always the same pulsotype nor the same antibiotic resistance profile. For example, four different swabs were taken from pig 141 (15 weeks old) on farm A. The isolate from the nares was slightly different from the isolate of the skin, perineum and rectum. Only the isolate from the nares and the perineum, two different pulsotypes, had the same antibiotic resistance profile. On farm B, pig 8173 was colonized on the ear and in the rectum with two MRSA isolates, which showed a completely different pulsotype.

On farm B, the farmers' wife was colonized on both sampling days with the same MRSA pulsotype, which was also isolated from pig 8173 ( 7.5 weeks old) on the first sampling day although they had different antibiotic resistance profiles (Figure 2). On the first sampling day, another pulsotype was isolated from the farmer's nose than on the second sampling day, and both pulsotypes were different from the one isolated from his wife.

\section{Discussion}

In this preliminary study we investigated two MRSA positive farms in detail in order to better understand farm epidemiology. Although we did not confirm the obtained MRSA isolates as MRSA ST398, we can assume these do in fact belong to the emerging livestock-associated MRSA type as none of these pig isolates were typeable by PFGE using SmaI as restriction enzyme. This is characteristic for MRSA ST398. 5

This study demonstrates that on positive farms, MRSA is widely spread among the pigs and in the environment. In total, $83 \%$ of the tested pigs were colonized. The high isolation rate reported in this study and other studies $^{20,21}$ could be explained by the fact that the pigs are grouped together and live in close contact, facilitating the spread of MRSA. MRSA was isolated from all pig age groups, ranging from one week to a few years (sows). Even

Table 3. Antibiotic resistance (in percentage) for 140 tested pig MRSA isolates from the two farms (farm A and Farm B) with corresponding 95\% CI and Odds Ratio's (OR), in the last column the P-value is given.

\begin{tabular}{|c|c|c|c|c|}
\hline & & sistant strains & & \\
\hline Antibiotics & Farm A $(95 \%$ CI $)$ & Farm B $(95 \%$ CI $)$ & OR $(95 \%$ CI $)$ & $\mathbf{P}$ \\
\hline CIP & $2.8 \%(0.8-9.6]$ & $1.5 \%(0.3-8.0]$ & $0.53(0.05-5.99)$ & 1 \\
\hline CHL & $0 \%(0-5.1)$ & $1.5 \%(0.3-8.0)$ & $3.27(0.13-81.68)$ & 0.234 \\
\hline ERY & $51.4 \%(40.1-62.6)$ & $67.2 \%(55.3-77.2)$ & $1.94(0.97-3.85)$ & 0.084 \\
\hline FUC & $0 \%(0-5.1)$ & $1.5 \%(0.3-8.0)$ & $3.27(0.13-81.68)$ & 0.234 \\
\hline GEN & $6.9 \%(3.0-15.3)$ & $97.0 \%(89.8-99.2)$ & 435.50 (81.58-2324) & $<0.0001$ \\
\hline KAN & $5.6 \%(2.2-13.4)$ & $94.0 \%(85.6-97.7)$ & $267.80(64.23-1116)$ & $<0.0001$ \\
\hline LIN & $98.6 \%(92.5-99.8)$ & $70.2 \%$ (58.3-79.8) & $0.03(0.004-0.26)$ & $<0.0001$ \\
\hline LZD & $0 \%(0-5.1)$ & $0 \%(0-5.4)$ & $1.07(0.02-54.89)$ & 1 \\
\hline MUP & $0 \%(0-5.1)$ & $0 \%(0-5.4)$ & $1.07(0.02-54.89)$ & 1 \\
\hline RIF & $0 \%(0-5.1)$ & $0 \%(0-5.4)$ & $1.07(0.02-54.89)$ & 1 \\
\hline SULF & $5.6 \%(2.2-13.4)$ & $14.9 \%(8.3-25.3)$ & $2.98(0.89-10.02)$ & 0.091 \\
\hline Q-D & $31.9 \%(22.3-43.4)$ & $7.5 \%(3.2-16.3)$ & $0.17(0.06-0.49)$ & $<0.001$ \\
\hline TET & $100 \%(94.9-100)$ & $100 \%(94.6-100)$ & $0.93(0.02-47.58)$ & 1 \\
\hline TOB & $5.6 \%(2.2-13.4)$ & $94.0 \%(85.6-97.7)$ & $267.8(64.2-1116)$ & $<0.0001$ \\
\hline TMP & $100 \%(94.9-100)$ & $100 \%(94.6-100)$ & $0.93(0.02-47.58)$ & 1 \\
\hline TYL & $51.4 \%(40.1-62.6)$ & $70.2 \%$ (58.3-79.8) & $2.22(1.11-4.47)$ & 0.026 \\
\hline
\end{tabular}

CIP, ciprofloxacin; CHL, chloramphenicol; ERY, erytrhomycin; FUC, fucidin; GEN, gentamicin; KAN, kanamycin; LIN, lincomycin; LZD, linezolid; MUP, mupirocin; RIF, rifampicin; SULF, sulfonamid; Q-D, quinupristin-dalfopristin; TET, tetracyclin; TOB, tobramycin; TMP, trimethoprim; TYL, tylosin. 

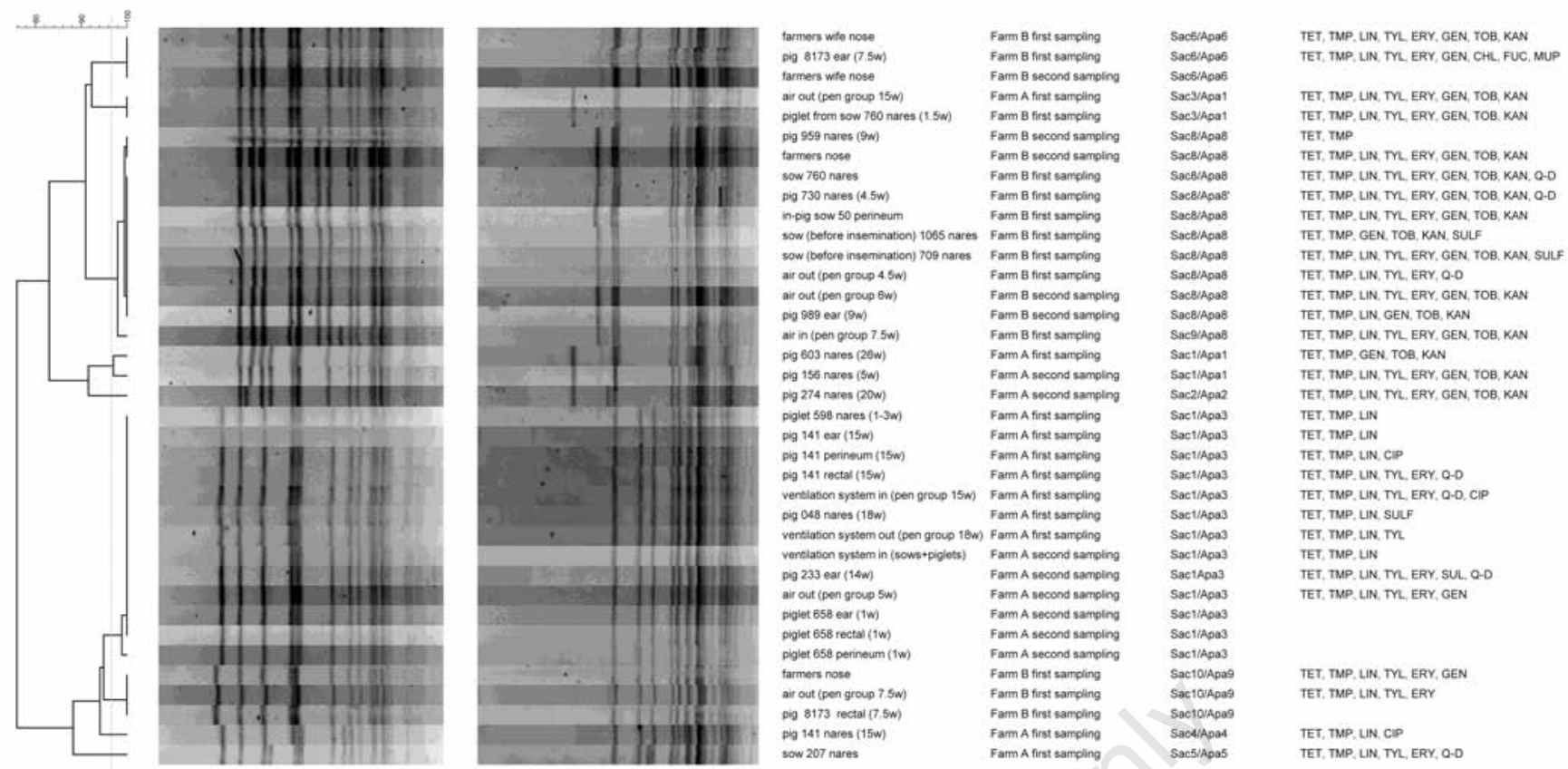

Figure 2. Consensus matrix and PFGE fingerprints from some selected MRSA isolates originating from both farms. The first colomn shows the origin of the isolate (including the age if the sample was from a pig), the second column the farm and the sampling time, the third column shows the pulsotype for both restriction enzymes and the fourth column shows the antibiotic resistance profile. Abbreviatons of the antibiotics can be found in Table 2. The grey line indicates the delineation level of $97 \%$.

piglets of one week old already carried MRSA. On both farms, the colonization degree of the piglets of 1-4 weeks old and the sows was similar. MRSA colonization of newborn piglets may occur by contact with the colonized mother sow or with a contaminated environment of the maternity ward. In this study we found indications of both sources of contamination, as the same and different MRSA strains were found on the sow and her non-weaned piglets. When weaning, the proportion of MRSA colonized animals rose, peaked at the age of 4-12 weeks, then decreased again. The reasons for this are unclear but may be related to stress at the point of weaning and the contact with piglets from other sows in the growing unit. On the other hand, colonization with resistant bacteria in younger animals has been shown before $^{22}$ and the observed variation may be related to a more general phenomenon that younger animals are more commonly colonized with resistant bacteria. The dynamics of primary colonization of piglets requires further investigation in order to be able to develop strategies for the remediation of MRSA on MRSA colonized farms.

MRSA was isolated from the nares, the skin, the perineum and the rectum of the pigs. A nasal swab was shown here to be the best sampling place to detect the MRSA status of a pig. However, by increasing the number of sampling sites, the chance of identifying MRSA positive pigs also increases. Furthermore, pigs may be colonized with different MRSA strains at the same time. Therefore, in order to determine the population variability and colonization potential of the MRSA strains, it can be useful to take different samples from one pig.

MRSA was recovered from all sampled surfaces on both farms. MRSA occurred on floors, walls, drinking nipples and in the ventilation system. In addition, the air was found to be contaminated. Air and dust can act as a possible vector of MRSA outside the pig barn. The recovery of MRSA at the outside of the ventilation system indicates that MRSA may be spread by air to other units on the farms or farms in the neighborhood. However, it is unclear to which distance dust contaminated with MRSA can travel and successfully contaminate other farms.

As shown before in more extended studies, 16,20 the transmission of MRSA between pig and humans may occur. The MRSA types isolated from the farmer and his family were also found in pigs. Although we had only a few isolates, we were able to demonstrate that persons may be colonized with two different strains, as one farmer was colonized with two different MRSA strains for the two sampling days. As we only isolated one colony from the farmer on each sampling day, it is possible that this farmer was colonized with both strains at the same time. Another possibility is that one MRSA strain replaced the other strain. A third option is that the original MRSA strain gained additional resistance genes.

All MRSA isolates were resistant to tetracy- cline, which is characteristic for MRSA ST398.23 Furthermore, all isolates were resistant to trimethoprim, which seems more specific for Belgium as other Belgian studies also report a high trimethoprim prevalence ${ }^{11,24}$ and contrasts with other studies where the prevalence is intermediate. ${ }^{25}$ It can be hypothesized that the trimethoprim resistence in this study is physically linked to the tetracycline resistance as described by Kadlec et al. ${ }^{26}$ However, this requires further study to identify the genes responsible for the resistance to tetracycline and trimethoprim. As all isolates were susceptible to the antibiotics rifampicin, mupirocin and linezolid, which are strictly used in human medicine, this does not compromise treatment of MRSA infected patients.

Antibiotic resistance could not be related to the antibiotic use on the studied farms. For example, in the case of the high number of MRSA isolates resistant to gentamicin, kanamycine, and tobramycine on farm B, no aminoglycosides were used in the six months before the actual sampling. However, it is possible that aminoglycosides were administered in the years before the study as we recorded the antibiotics use only in the 6 months before the samplings. Furthermore, resistance to these three antibiotics is often conferred by the $\operatorname{aac}\left(6^{\prime}\right)-\operatorname{aph}\left(2^{\prime \prime}\right)$ gene, which is located on a transposon and widely distributed. So, once this transposon is introduced on a farm, for example via purchased animals, it can spread fast on the farm. 
Although only a limited number of isolates were further characterized, we demonstrated that several different strains circulate on one farm at one time. More specifically, even within pens with pigs from the same age, even within the nursery unit of one sow and her piglets of one week old and even within one pig, different pulsotypes and different antibiotic resistance profiles were found. This has consequences for further research. For example, when studying the epidemiological routes and sources on a MRSA positive farm, researchers must take care to take enough samples.

In conclusion, on MRSA colonized farms, a high percentage of the pigs in all age groups are colonized with MRSA. MRSA prevalence increases especially after weaning. To detect the status of a pig, the best sampling site is the nares, although it is recommended to take two different samples, including the nares. The barn environment, including the air is often contaminated with MRSA, analogous to the pigs' colonization. Different MRSA pulsotypes and different antibiotic resistance profiles circulate on a MRSA positive farm. Further studies are necessary to clarify the origin and the spread of this LA-MRSA in order to formulate measures on the prevention of MRSA on pig farms.

\section{References}

1. Leonard FC, Markey BK. Methicillin-resistant Staphylococcus aureus in animals: a review. Vet J 2007;185:155-9.

2. Deurenberg RH, Vink C, Kalenic S, et al. The molecular evolution of methicillinresistant Staphylococcus aureus. Clin Microbiol Infect. 2007;13:222-35.

3. Voyich JM, Otto M, Methema B, et al. Is panton-valentine leukocidin the major virulence determinant in community-associated methicillin-resistant Staphylococcus aureus disease. J Infect Dis 2006;194: 1761-70.

4. van Duijkeren E, Ikawaty R, BroekhuizenStins MJ, et al. Transmission of methicillin-resistant Staphylococcus aureus strains between different kinds of pig farms. Vet Microbiol 2008;126:383-9.

5. Voss A, Loeffen F, Bakker J, et al. Methicillin-resistant Staphylococcus aureus in pig farming. Emerging Infect Dis 2005;11:1965-6.

6. Morgan M. Methicillin-resistant Staphylococcus aureus and animals: zoonosis or humanosis? J Antimicrob Chemother 2008;62:1181-7.

7. Vanderhaeghen W, Hermans K, Haesebrouck F, Butaye P. Methicillin-resistant Staphylococcus aureus (MRSA) in food production animals. Epidemiol Infect 2010;138:606-25.

8. O'Mahony R, Abbott Y, Leonard FC, et al. Methicillin-resistant Stapylococcus aureus (MRSA) isolated from animals and veterinary personnel in Ireland. Vet Microbiol 2005;109:285-96.

9. Lee JH. Methicillin (Oxacillin)-resistant Staphylococcus aureus strains isolated from major food animals and their potential transmission to humans. Appl Environ Microbiol 2003;69:6489-94.

10. Persoons D, Van Hoorebeke S, Hermans K, et al. Methicillin-resistant Staphylococcus aureus in poultry. Emerging Infect Dis 2009;15:452-3.

11. Willems G, Dispas M, Denis 0, et al. Characterization of MRSA from pigs in Belgium. 2nd Symposium on Antimicrobial Resistance in Animals and the Environment (AREA), 2007, Tours, France.

12. van Loo I, Huijsdens XW, Tiemersma E, et al. Emergence of methicillin-resistant Staphylococcus aureus of animal origin in humans. Emerging Infect Dis 2007;13: 1834-9.

13. Denis 0, Suetens C, Hallin M, et al. Methicillin-resistant Staphylococcus aureus ST398 in swine farm personnel, Belgium. Emerging Infect Dis 2009;15: 1098-101.

14. Moodley A, Nightingale EC, Stegger M, et al. High risk for nasal carriage of methicillin-resistant Staphylococcus aureus among Danish veterinary practitioners. Scand J Work Environ Health 2008;34:1517.

15. Moodley A, Stegger M, Bagcigil AF, et al. Spa typing of methicillin-resistant Staphylococcus aureus isolated from domestic animals and veterinary staff in the UK and Ireland. J Antimicrob Chemother 2006;58:1118-23.

16. van den Broek IVF, van Cleef BAGL, Haenen A, et al. Methicillin-resistant Staphylococcus aureus in people living and working in pig farms. Epidemiol Infect
2008;24:1-9.

17. Strandén A, Frei R, Widmer AF. Molecular typing of methicillin-resistant Staphylococcus aureus: can PCR replace pulsedfield gel electrophoresis? J Clin Microbiol 2003;41:3181-6.

18. Maes N, Magdalena J, Rottiers S, et al. Evaluation of a triplex PCR assay to discriminate Staphylococcus aureus from coagulase-negative staphylococci and determine methicillin resistance from blood cultures. J Clin Microbiol 2002;40: 1514-7.

19. Rasschaert G, Vanderhaeghen W, Dewaele I, et al. Comparison of fingerprinting methods for typing methicillin-resistant Staphylococcus aureus sequence type 398 . J Clin Microbiol 2009;47:3313-22.

20. Khanna T, Friendship R, Dewey C, Weese JS. Methicillin resistant Staphylococcus aureus colonization in pigs and pig farmers. Vet Microbiol 2008;128:298-03.

21. Huijsdens XW, van Dijke BJ, Spalburg E, et al. Community-acquired MRSA and pigfarming. Ann Clin Microbiol Antimicrob 2006;5:26.

22. Mathew AG, Upchurch WG, Chattin SE. Incidence of antibiotic resistance in fecal Escherichia coli isolated from commercial swine farms. J Anim Sci 1998;76:429-34.

23. De Neeling AJ, van den Broek MJM, Spalburg EC, et al. High prevalence of methicillin resistant Staphylococcus aureus in pigs. Vet Microb 2007;122:36672.

24. Verhegghe M, Pletinckx LJ, Crombé F, et al. Screening of 30 Belgian pig farms and pig farms with other livestock for the presence of methicillin-resitant Staphylococcus aureus (MRSA) ST398. International Symposium on Staphylococci and Staphylococcal Infections, 2010, Bath, UK.

25. Kadlec K, Ehricht R, Monecke S, et al. Diversity of antimicrobial resistance pheno- and genotypes of methicillinresistant Staphylococcus aureus ST398 from diseased swine. J Antimicrob Chemother 2009;64:1156-64.

26. Kadlec K, Schwarz S. Identification of a novel trimethoprim resistance gene, dfrK, in a methicillin-resistant Staphylococcus aureus ST398 strain and its physical linkage to the tetracycline resistance gene tet(L). Antimicrob Agents Chemother 2009;53:776-8. 